\title{
Characterization of Third-Harmonic Target Plan and Irradiance on the National Ignition Facility Beamlet Demonstration Project
}

Paul J. Wegner, Bruno M. Van Wonterghem, Shamasunder N. Dixit, Mark A. Henesian, Charles E. Barker, Calvin E. Thompson, Lynn G. Seppala and John A. Caird

This paper was prepared for submittal to the

12th Topical Meeting on the Technology of Fusion Energy

Reno, NV

June 16-20, 1996

April 25, 1997

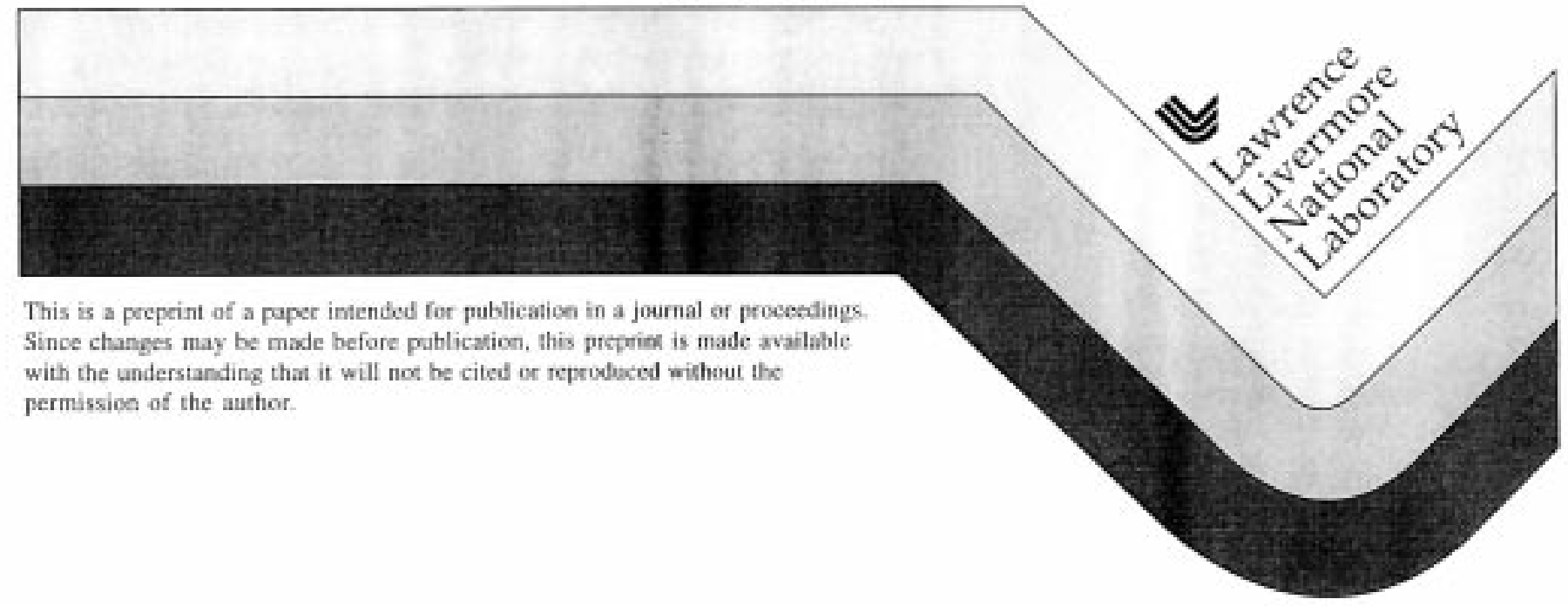




\section{DISCLAIMER}

This document was prepared as an account of work sponsored by an agency of the United States Government. Neither the United States Government nor the University of California nor any of their employees, makes any warranty, express or implied, or assumes any legal liability or responsibility for the accuracy, completeness, or usefulness of any information, apparatus, product, or process disclosed, or represents that its use would not infringe privately owned rights. Reference herein to any specific commercial products, process, or service by trade name, trademark, manufacturer, or otherwise, does not necessarily constitute or imply its endorsement, recommendation, or favoring by the United States Government or the University of California. The views and opinions of authors expressed herein do not necessarily state or reflect those of the United States Government or the University of California, and shall not be used for advertising or product endorsement purposes. 


\title{
CHARACTERIZATION OF THIRD-HARMONIC TARGET PLANE IRRADIANCE ON THE NATIONAL IGNITION FACILITY BEAMLET DEMONSTRATION PROJECT
}

\author{
Faul J. Wegner, Bruno M. VanWonterghem, Shamasunder N. Dixit, Mark. A. Henesian, \\ Charles E. Barker, Calvin. E. Thompson, Lynn. G. Seppala and John A. Caird
}

\author{
Lawrence Livermore National Laboratory \\ PO Box 808, L- 487 \\ Livermore, CA 94550 \\ (510) 423-2483
}

\begin{abstract}
The Beamlet laser is a single-aperture prototype for the National Ignition Facility (NIF), We have recently installed and activated a $55 \mathrm{~m}^{3}$ vacuum vessel and associated diag. nostic package at the output of the Beamiet that we are using to characterize target plane irradiance at high power. Measurements obtained both with and without a kinoform diffractive optic are reported. Dependences on critical laser parameters including output power. spatial filtering. and wavefront correction afe discussed and compared with simulations.
\end{abstract}

\section{INTRODUCTION}

ICF target designs for the NIF demand high-quality beams from the laser driver. A typical indirece drive target for [CF consists of a fuel capsule centered in a can-shaped hohlraum; beams from the driver enter the target through laser entrance holes (LEH's) located on each end of the can.' The LEH's are designed to be small- on the order of $3 \mathrm{~mm}$ or lessto maintain a uniform irradiation envifonment for the capsule and to minimize radiation transport losses from the hohlraum. Nevertheless, plasma blow-off from the edges of the LEH must not elip the bearns, even at late times in the drive polse. therefore the beams must be focused to a diameter that is substantially smallet than that of the LEH. A further requirement is that the laser irradiance on the walls of the hohlraum be smooth and devoid of hot spots that would otherwise drive plasma filamentation and stimulated scattering processes. and reduce the efficiency of the implosion. ${ }^{2}$ Therefore, there is a need to tailor the spatial profile of the focused drive beam and apply some form of temporal smoothing. These conditions are to be met at a total drive energy of $1.8 \mathrm{MJ}$ and a peak power of $500 \mathrm{TW}$, which it is estimated will produce ignition with a safety margin of two. ${ }^{3}$ For the 192 beamlets of the
NIF, this translates into an energy of $9.4 \mathrm{~kJ}$ and a peak power of $2.6 \mathrm{TW}$ per beamilet.

In this paper we discuss measurements performed on the Beamlet Laser Facility at Livermore to characterize target plane irradiance at the power levels expected for the NIF. We compare our results to the NIF specifications, and idenufy the critical laser parameters impacting performance that will enable us to extrapolate these results from Beamlet to the NIF

\section{TARGET REQUIREMENTS FOR LASER IRRADLANCE}

The design requirements for the NIF include the follow. ing criteria for the focused laser spot. Focal spos characteristics other than those described here have been requested by a number of NIF user groups, however the emphasis here will be on the baseline requirements for the NIF as specified in the system design requirements. The design energy of $1.8 \mathrm{MJ}$ and the design power of $500 \mathrm{TW}$ is to be delivered at a wavelength of $0.351 \mu \mathrm{m}$ inside a 600 - $\mu \mathrm{m}$ diameter spot (half-angle of $43 \mu$ rad for the NIF 7-meter focus lens). The spot profile is to approximate an eighth-power super-Gaussian with $1 / \mathrm{e}$ radius of no moxe than $250 \mu \mathrm{m}$

$$
l\langle r\rangle=I_{0} \exp \left[-\left(r / r_{0}\right)^{8}\right], \quad r_{0} \leq 250 \mu \mathrm{m}
$$

As a radius of $1.5 \mathrm{~mm}$ corresponding to the nominal size of the target LEH, the irradiance is to be less than $10^{14} \mathrm{~W} / \mathrm{cm}^{2}$. Beam smoothing requirements for the spor profile have not yet been tested on the Beamlet and will non be discussed in detail in this paper. The requirements call for the focal spon to be temporally smoothed using one-dimensional dispersion ot 


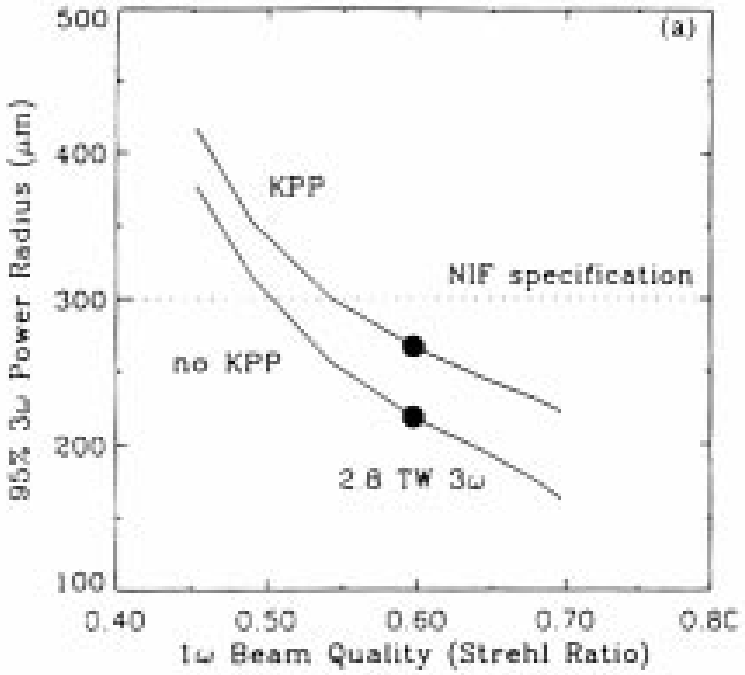

Figure 1. (a) Calculation based on laser simulations showing the spoe size of the $3 \omega$ beam versus the I $\omega$ beam quality from the laser amplifier. Upper and lawer curves correspond to simulations with and without beam conditioning from a kinoform phase plate. Cor. responding focal plane distributions calculated for an output power of $2.8 \mathrm{TW}$ are shown in (b) and (c). Displayed regions span $\pm 600 \mathrm{\mu m}$

3 angstroms of laser bandwidth, critically dispersed. ${ }^{4}$

\section{LASER CONTROL PARAMETERS}

Calculations indicate that the above criteria can be met provided the quality of the beam from the driver is maintained at a high level. Figure 1 shows results from fullaperture laser simulations of the Beamlet which calculate the

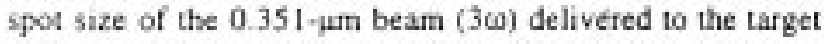
versus the the quality of the $1.053-\mu \mathrm{m}$ bearn (19) at the output of the laser amplifier. The lower curve is calculated for the case of no beam conditioning; the upper curve for the case when the $3 \omega$ beam is conditioned with a diffractive optic called a kinoform phase plate (KPP). The purpose of the KPP is to modify the aberrated focal spot produced by the laser to produce a more homogeneous irradiance distribution in the form of a speckle field with a spatial envelope that closely approximates the required eighth-power super Gaussian." The price paid for the improvement in distribution is an in-

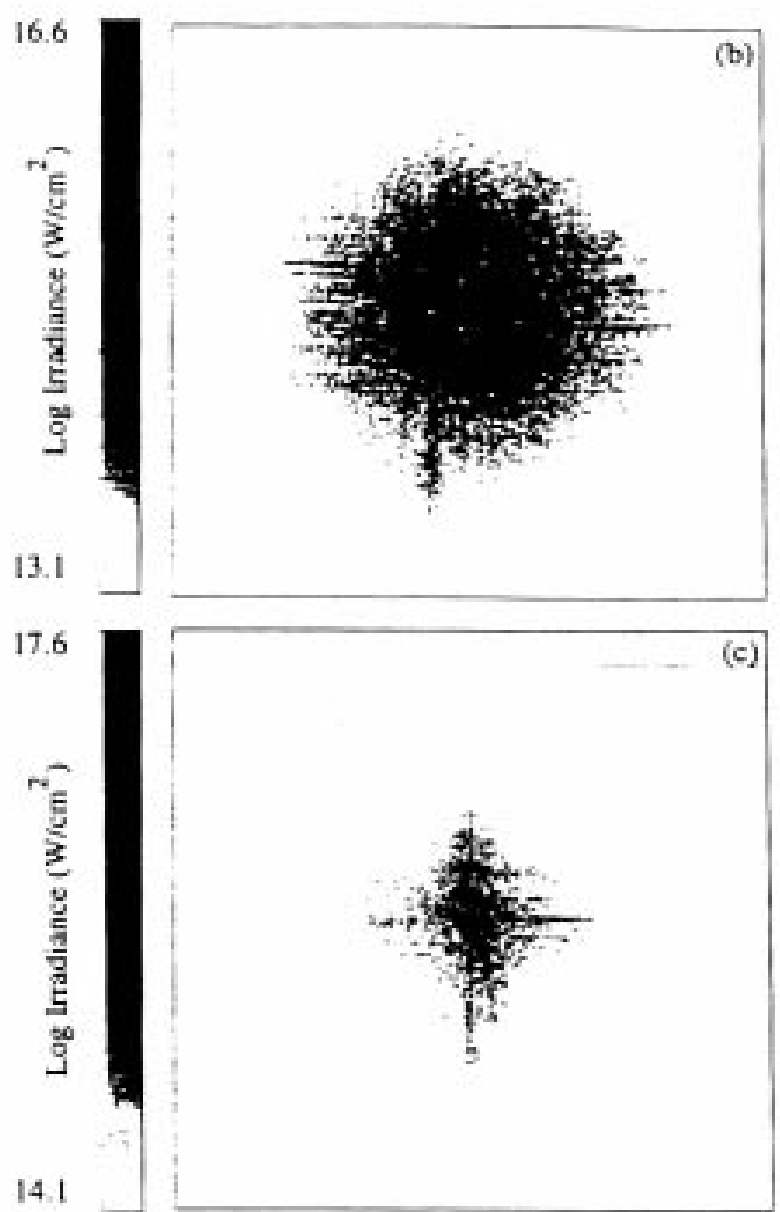

crease in the spot size; nevertheless the simulations indicate that at an output power of $2.8 \mathrm{TW}(3 \omega)$ we should be able to meet the NIF specification of $2.6 \mathrm{TW}$ inside of $600 \mu \mathrm{m}$.

There are several laser performance issues that affect beam quality and determine whether we can in fact meet the NIF specifications. First, there are low-frequency thermally induced wavefront distortions originating in the laser amplifief that must be corrected with a deformable mirror. ${ }^{6}$ Figure 2a shows a calculation of the wavefront at the output of the Beamlet for the case of no wavefront correction; the \pm 1 wave of distortion shown in the figure is caused by pump-induced steering effects in the amplifier slabs. When correction is applied with a deformable mirror having the surface figure shown in Figure 2b, the output wavefront is calculated to be nominally flat and the $1 \omega$ Strehl ratio is increased from 0.1 to $>0.7$. The mirror in these simulations is a model of the 39 . actuator device currently in use on the Beamlet.' The angular band width of the Beamlel mirror allows for the correction of 


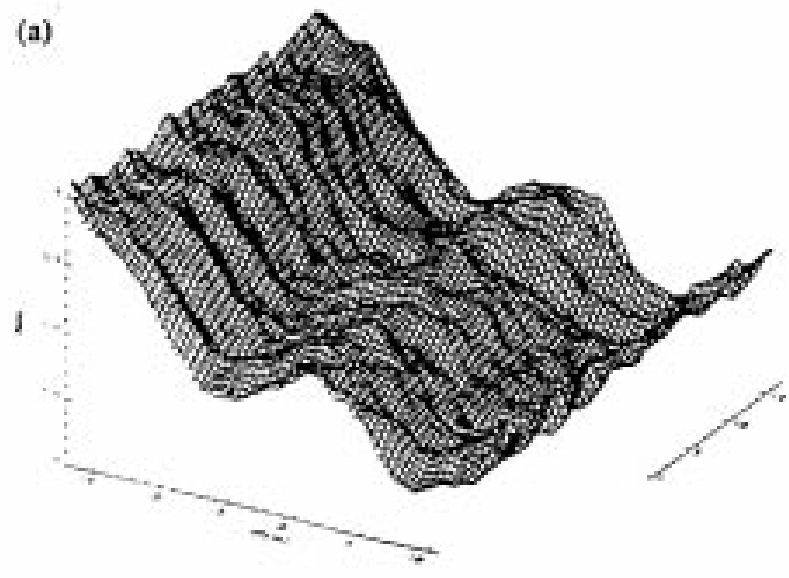

(b)

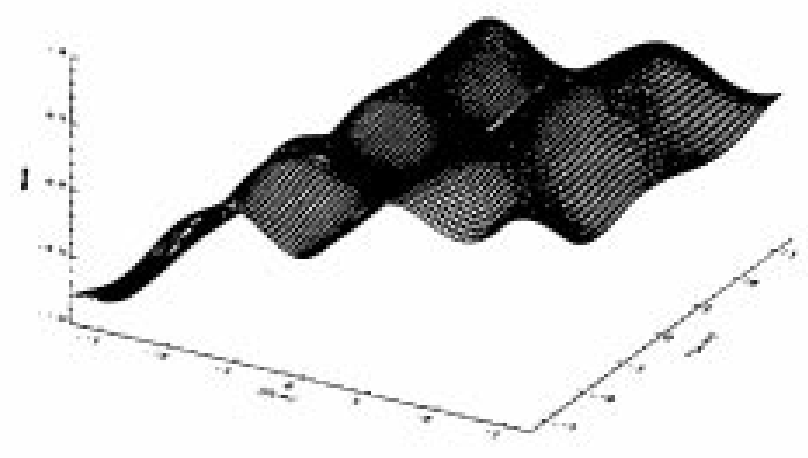

(c)

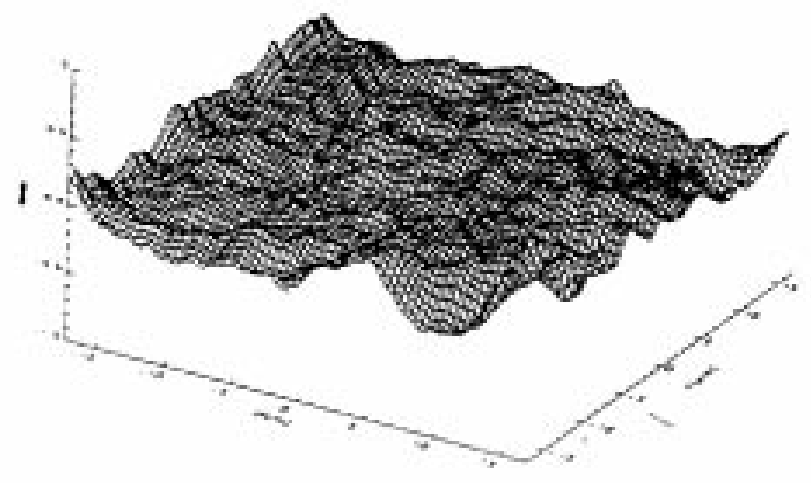

Figure 2. Simulations demonstrating the correction of long scalelength wavefront distortions with a deformable mirror. (a) Pump induced wavefront distortions reduce the ootput $1 \mathrm{\omega}$ Strehl ra. tio to $<0.12$. (b) Surface figure of 39 -acnuan deformable minot for wavefront correction. (c) Corrected low.power output wavefront with Strehl ratio of $>0.7$. wavefront etrors ranging up to approximately \pm 20 urad

Of additional concern are the amplitude and wavefront distortions in the beam that oceur at frequencies exceeding the practical bandwidth of a deformable mirror. Angular bandwidth associated with high-frequency ripple can actual. ly grow as a result of propagation instabilities in the laser. The mechanism for growth is the intensity-dependent refractive index in the laser components, a manifestation of the third-order Kerr effect, which enables a weak ripple wave co-propagating with a strong pump wave to couple and scatter a fourth wave, conjugate in angle to the ripple (see Figure $3 a){ }^{1-10}$ Subsequent interaction of the conjugate wave with the pump generates another ripple wave, and so on, in a process which if left unchecked, leads ultimately to the generation of higher-order ripple modes, advanced self focussing and beam breakup.

Ripple growth poses a problem well before the beambreakup stage because it is a direct means by which energy can be coupled into high-angle modes that focus outside of the target. In the perturbation regime, the ripple field can be shown to evolve through a given component as $\exp [ \pm \Omega t]$. with $\Omega$ depending on the angle of the ripple wave according ம

$$
\Omega(\theta)=\frac{\theta}{\theta_{m}} \sqrt{2-\left(\frac{\theta}{\theta_{m}}\right)^{2}} B_{0}, \quad B_{0}=\frac{2 \pi n}{\lambda} \gamma l
$$

Here $l$ is the background intensity of the pump field, $n$ is the refractive index, $\lambda$ the wavelength and $\gamma$ the nonlinear coefficient in the material. At the optimum ripple growth angle denoted by $\theta_{m}$

$$
\theta_{m}=\sqrt{\frac{2 \eta I}{n}}
$$

power in the ripple mode grows as $\exp (2 B)$ with $B=B_{02}$ be . ing the familiar notation for the nonlinear phase shift incurred by the ripple wave. On systems with many components of various spacings, the actual power gain and its dependence on ripple angle can be quite complex," as demonstrated by Figure 3b, which plots the calculated ripple growth spoctrum for the Beamlet laser at a $3 \omega$ output power of $3.5 \mathrm{TW}$. The lower curve, corresponding to the to spectrum, was generated by injecting a field with perturbative noise terms uniformly distributed in phase and amplitude into a model of the laser amplifier and calculating the gain at the output as a function of ripple angle. The large peak visible at 200 yrad reflects the tendency for the beam to break up into 5-mm cells unless this particular growth mode is suppressed: 
(a)

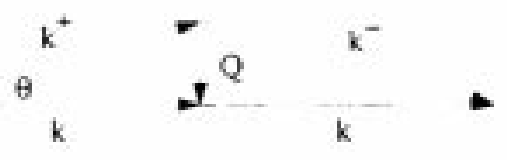

(b)

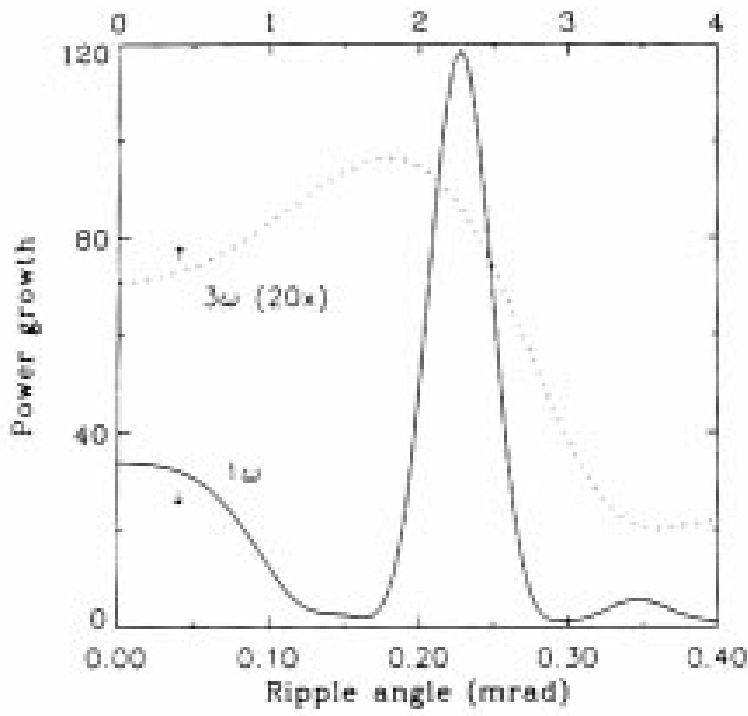

Figure 3. (a) Wave vector diagram of degenerate four-wave interaction responsable for nonlinear ripple growth in materials possessing a Kerr response Q. (b) Calculated $I \omega($ _ $)$ and 300 (....) ripple growath spectra for the Beamlet laser at a 3 eoulput power of 3.5 TW in $234-\mathrm{cm}$ beam. $3 \omega$ curve has been scaled by a factor of 20 .

thus the largest pinhole size used in the Beamlet spatial filters corresponds to a cutoff angle of $\pm 200 \mu \mathrm{rad}$. The upper curve corresponds to the 30 growth spectrum calculated for the final optics. In this case. maximum growth occurs at angles in the vicinity of 2 mrad (ripple scale lengths on the order of 100 to $200 \mu \mathrm{m}$ ) and the potential for $\mathrm{a} 5 \mathrm{x}$ increase in power scat. tered to these angles is indicated

Controls for ripple growth are limited, but critical for the production of well- focused beams on both the Beamlet and the NIF. Optical components for these facilities must comply with stringent specifications for homogeneity and surfacefinish specifically to limit the source terms for this effect. Pinhole staging in the spatial filters, and the $B$ incurred between and after the filters are both important control parameters for the beam characterization studies discussed below.

\section{BEAMLET FOCAL PLANE DIAGNOSTICS}

The Beamlet laser is a single-aperture prototype of the NIF design that was built to demonstrate the laser science and technology of the NIF arehitecture. ${ }^{12}$ It incorporates a fourpass cavity contaning eleven Nd:Phosphate glass amplifier slabs, a $37 . \mathrm{cm}$ aperture plasma-electrode Pockels cell/polarizer switch to eject the beam from the cavity, a fiveslab booster amplifier, and a $37-\mathrm{cm}$ aperture frequency converier comprised of a type I KDP doublet and type II KD*P

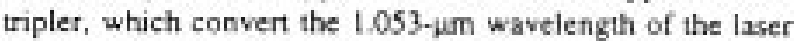
to the 0.351 um wavelength delivered to the target. Two 18 . meter spatial filters image-relay the system and control an. gular bandwidth: one filter relays the cavity, and the second transpons the beam from the booster amplifiers to the frequency converter. The 39-actuator deformable mirtor resides in the injection optics assembly near the pinhole plane of the cavity filter.

For characterizing the irradiance in the target plane we recently installed a $55 \mathrm{~m}^{3}$ vacuum vessel and associated diagnostic package at the output of the Beamlet, as sbown in Figure 4. A square fused silica lens of 7-meter focal length forms a vacuum barrier for the vessel and produces a $3 \omega \mathrm{fo}$. cus at a plane equivalent to the target location on the NIF. An off-axis lens design. per the original NIF baseline, yields an equivalent wedge angle of $4.5^{\circ}$ which we use to angularly disperse the $3 \omega$ beam from the $1 \omega$ and $2 \omega(0.528 \mu \mathrm{m}$ wave. length) radiation left over from the frequency conversion process. Provisions exist for installing a debris shield of a KPP immediately downstream of the lens when required. The focus at the target plane is imaged and attenuated by a 1 . meter aperture telescope housed inside the vessel. The telescope is comprised of three uncoated spherical mirfors fabricated from fused sitica and configured to form an eccentric portion of a centered optical system, as illustrated in Figure $4 b$. One of the principle advantages of this design is its large working distance which makes it possible to expand the post focus beam up to a size of $60 \mathrm{~cm}$ and thus reduce the threat of damage to the primary mirroe. In addition, the design provides an unobscured aperture and is afocal, meaning that planes in the vicinity of the target plane are imaged with near-constant magnification. Auxiliary optics downstream of the telescope feed an extensive suite of energy/power detectors and imaging sensors.

The primary energy diagnostic is a full-aperture calorimeter located in the vessel near a relay plane of the final focus lens, which measures the total energy in the first, second and third harmonic wavelengths. Energy detectors for the individual wavelengths are calibrated by reference to this calonmeter. Additional detectors measure the $3 \omega$ energy in the target plane out to a radius of $20 \mathrm{~mm}$ ( $\pm 3 \mathrm{mrad}$ ). and the 
(a)

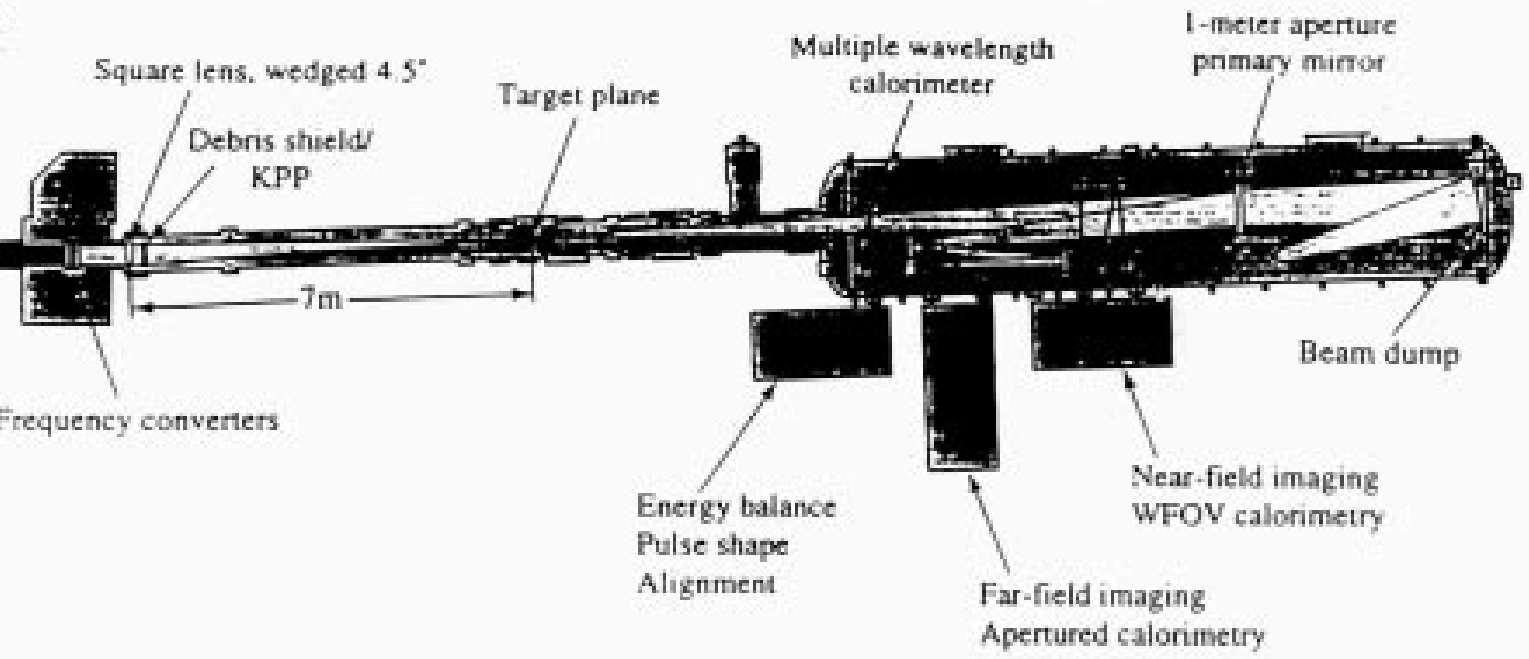

(b)

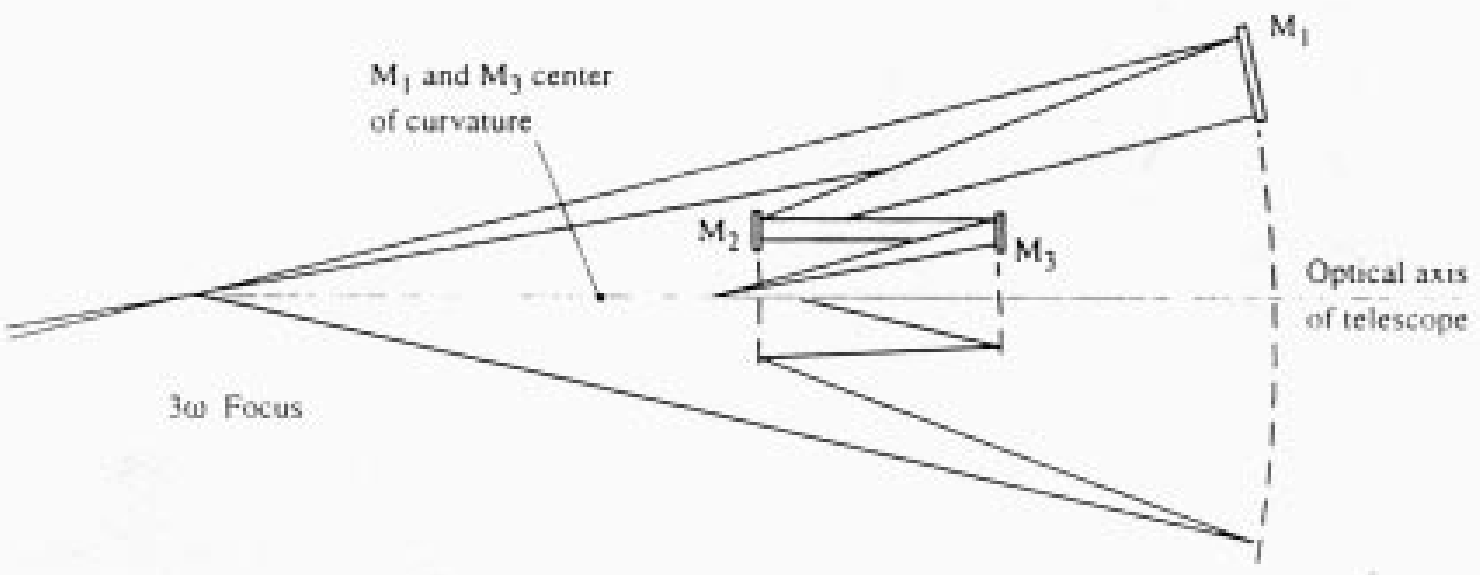

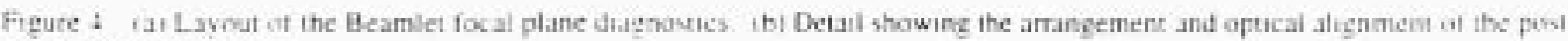
focus ielescupe mitrots 
(a)

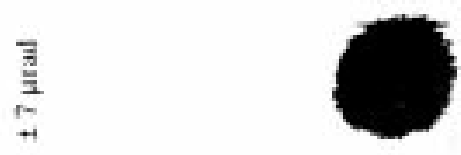

v

(b)

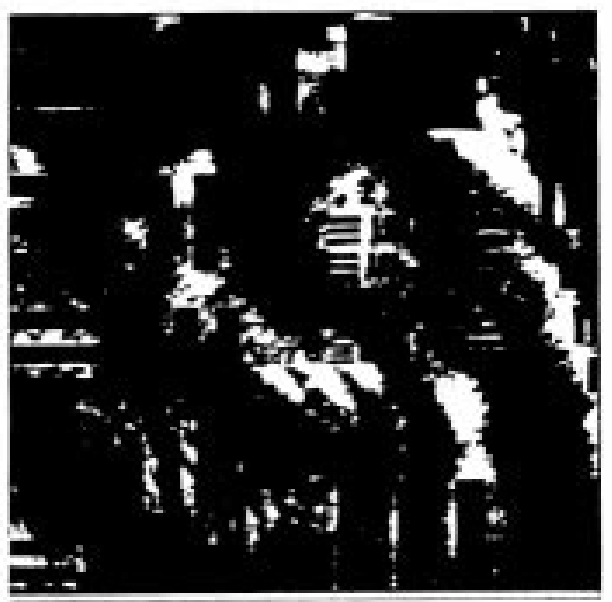

Figure 5 (a) Image of a point source situated at the target plane. $81.5 \%$ of the energy is within a spot size equal to 1.6 times the diffraction limit. (b) Image of a backlit test target located at the final focus lens, demonstratıng 70 um resolution.

fraction of this energy that passes through an aperture equivalent to the $3 \mathrm{~mm}$ LEH. Pulse shape is measured with a 4-ps resolution streak camera.

Imaging sensors include two target plane cameras with \pm 100 and \pm 300 urad fields of view, a multiple image camera which views several planes spaced by $4 \mathrm{~mm}$ about the target plane, and two nearfield cameras which measure both large scale ( $>1 \mathrm{~mm})$ and fine scale $(<100 \mu \mathrm{m})$ bearn structure at the final focus lens. Target-plane imaging is accomplished with neaf-diffraction limited resolution, as demonstrated by Figure 5, which shows the image of a $2 \omega$ fiber optic pount source ( $9 \mu \mathrm{m}$ diameter) situated at the 30 target plane. The measurement was performed at the second harmonic because of the irradiance levels needed to overcome the losses of the uncoaed telescope mirrors. Nearfield imaging capability has also been quantified. Figure $5 b$ is an image of a resolution test target situated at the final focus lens demonstrating a re. solving power of better than 14 lines per mm. This camera has only recently been activated and will be used to charac. terize the growth of high-frequency $3 \omega$ ripple in the final focusing opsics.

\section{MEASUREMENTS AND ANALYSIS}

The tests we are currently conducting on the Beamlet afe designed to evaluate beam quality during the most stressfut period of the ICF drive pulse. The baseline ICF requirements for the NIF call for a 20 ns duration pulse with a $50 x$ increase in power from front to back. By propagating short $200 \mathrm{ps}$ duration pulses through the laser under conditions equivalent to those expected at the peak of the drive pulse we are able to obtain a "snap-shot" of beam quality that would otherwise be masked by integration effects. To simulate the conditions of reduced gain and increased B integral seen by photons at the peak of the long saturating pulse we conducted the majonty of these tests with the booster amplifiers turned off.

Measurements of nearfield beam quality at high power show the irradiance distribution to be spatially uniform. Figure 6 is a CCD image of a 1.9 TW 30 beam obtained under near red-line conditions: $\Delta \mathrm{B}^{\prime}$ 's of 1.0 and 1.5 radians in the cavity and booster sections respectively, with \pm 200 urad pinboles installed in the cavity and transport spatial fitters. Restricting analysis of the irradiance distribution to the central $31-\mathrm{cm}$ portion of the beam (to omit edge effects) reveals

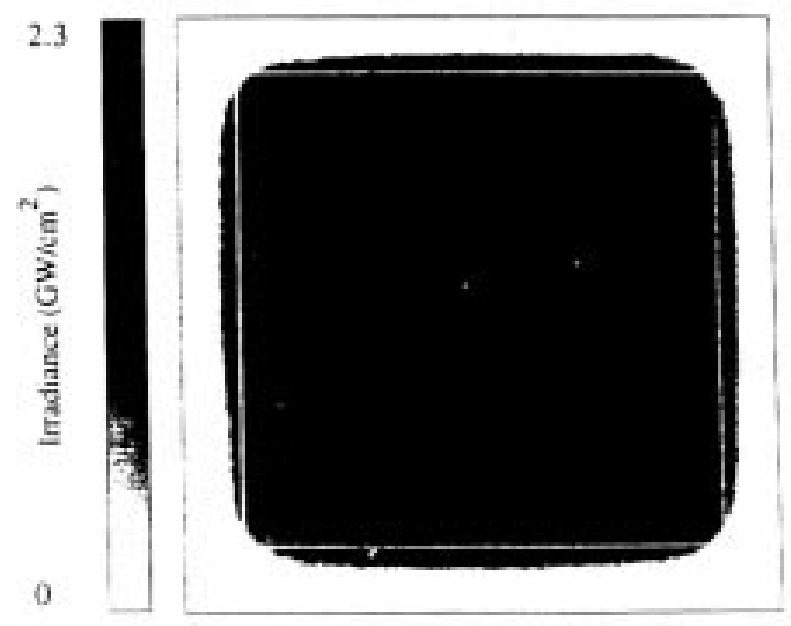

Figure 6. 1.9 TW 36 beam at the input to the final focus lens imaged with a $\mathrm{L}-\mathrm{mm}$ resolution CCD camera. $31-\mathrm{cm}$ subreguon analyzed for irradiance statistics is highlighted. 

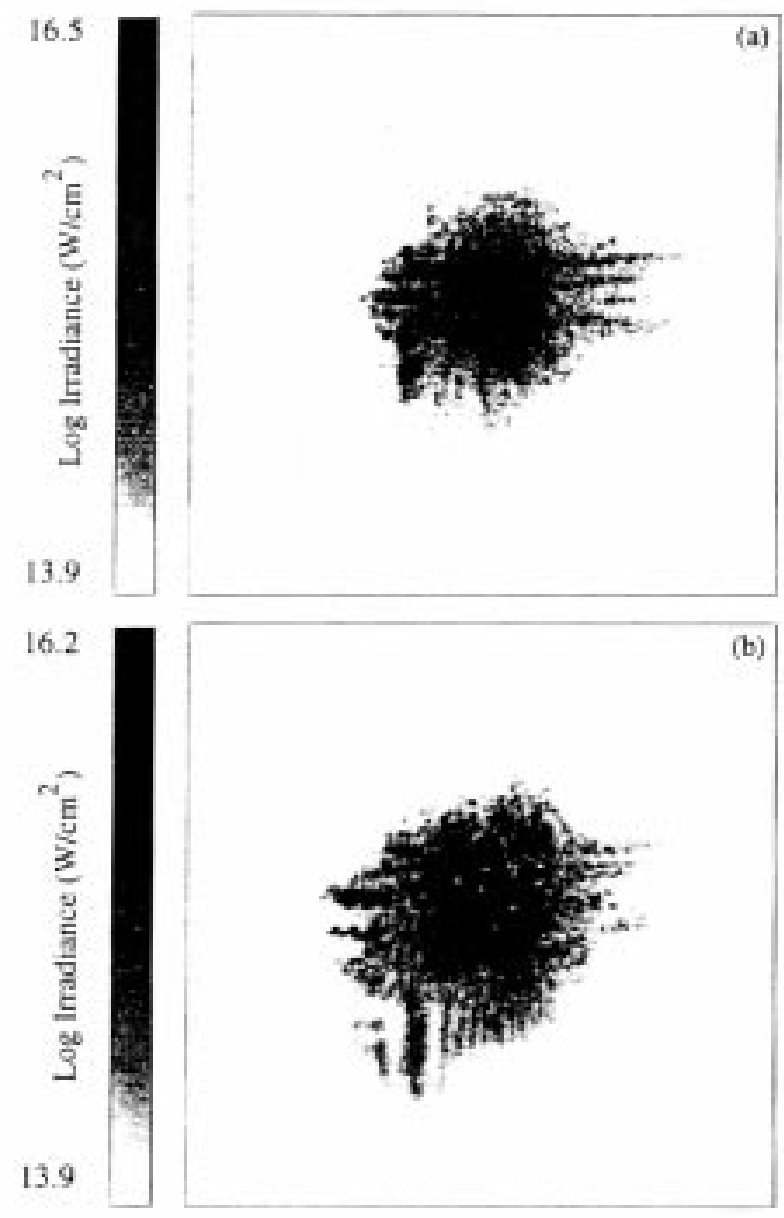

Figure 7. (a) 2.4 TW $3 w$ focal spot measured without the KPP. (b) Subsequent 2.1 TW $3 \omega$ focal spot showing increased distortion from thermally-induced wavefront aberrations in the amplifiers. Displayed regions span $\pm 600 \mu \mathrm{m}$.

a contrast, defined as the ratio of the standard deviation to the mean, of 0.11 , with a peak to mean modulation of better than 1.35 to 1 . Better compensation for spatial gain variations in the amplifiers ${ }^{\circ}$ would reduce the long scale-length irradiance non-uniformities visible in the image and improve the contrast further.

Farfield measurements confirm that characteristics of the target plane irradiance on a scale length of the LEH are dominated by thermal effects in the laser amplifiers. Figure $7 \mathrm{a}$ shows a high-resolution $\mathrm{CCD}$ image of the unconditioned Tu focal spor generated on a 2.4 TW shot. Radial integration of the image reveals that $80 \%$ of the power is contained inside a half angle of $19 \mu$ rad. This data was obtained on the first shot of the day when the amplifiers are comparatively cool. Build up of heat in the amplifiers over successive shots throughou the day generates thermal gradients and turbu. lence that affect beam quality, but which cannot be completely compensated for by the deformable mirror (currently the Beamlet mirror must be locked into position for a finite time period prior to each shot). Figure $7 b$ shows this effecs in the focal spot generated during the second shot of the day, in which at a reduced power of 2.I TW, the spot size containing $80 \%$ of the power was observed to increase by over $20 \%$. Amplifier heating is currently a problem for the Beamlet, but is not expected to be a concern for the NIF, in which active cooling is an integral part of the amplifier design. ${ }^{13}$

The focal spot generated with the KPP closely approach es the NIF baseline criteria for the irradiance distribution inside the L.EH. Representative data obtained on a 2.1 TW shot and shown in Figure 8 reveals that $95 \%$ of the laser energy entering the LEH is contained within a $600 \mu \mathrm{m}$ diameter spot. The 1/e radius of the spor profile measures less than 250 um and the irradiance levels at the edge of the LEH are below $10^{14} \mathrm{~W} / \mathrm{cm}^{2}$. Distortions of the spot profile are evident in the form of flare-like structures extending out beyond $40 \mu \mathrm{rad}$. This structure contains a few percent of the energy and combined with turbulence effects, prevents the achievement of a sharply delineated distribution (see Figure 9). The origin of this structure is under investigation but it is present in the unconditioned data as well, indicating that improvements in beam quality will most likely be needed to meet the superGaussian profile requirement. Preliminary measurements also indicate a power-dependent increase in the level of seat-

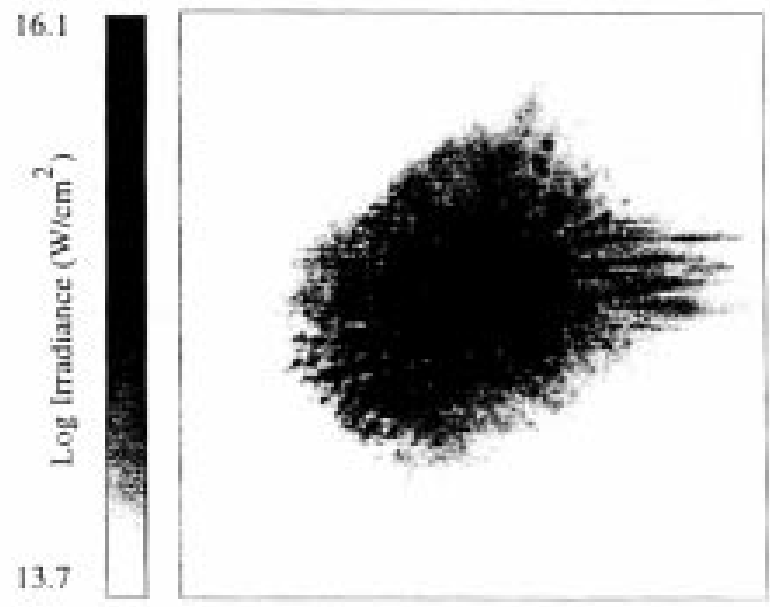

Figure 8. Measured 2.1 TW 3en focal spoe with the KPP. Displayed region spans $\pm 600 \mu \mathrm{m}$. 


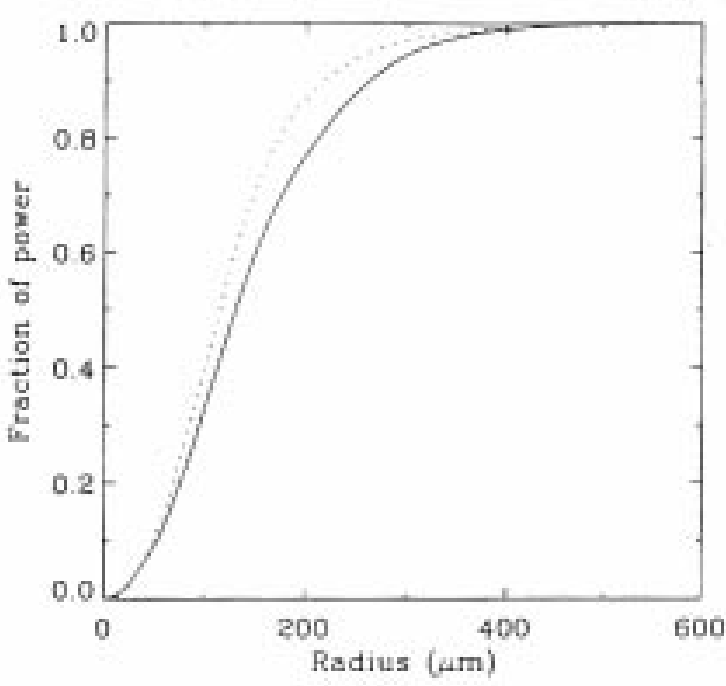

Figure 9. Radial distribution of power normalized to the total power inside the LEH for measured $2.1 \mathrm{TW}(\ldots$ ) and calculated $2.8 \mathrm{TW}$ (....) focal spot with beam conditioning. Model does not include tur. bulence effects in the amplifiers.

tered radiation lying outside of the LEH. An observed increase from $-2 \%$ at I TW to $-7 \%$ at 2.4 TW must be confirmed with additional measurements, but is not inconsistent with the calculation of Figure $3 \mathrm{~b}$.

\section{CONCLUSION}

This first series of Beamlet 3e focal spot characterization tests have come close to demonstrating the NIF requirements for laser irradiance in the target plane. Peak powers of up to 2.4 TW have been delivered through the final optics with no evidence of small-scale beam breakup or filamentation. This result has been accomplishod at an aperture size that is approximately ten percent smaller than that expected for the NIF.

Several issues remain for demonstrating NIF quality focal spots on the Beamlet. Wavefront distortions generated by heat build up in the laser amplifiers cause the spor size to increase on successive shots; active cooling of the Beamlet amplifiers, and closed-loop operation of the deformable mir. ror up until shot time are being pursued as potential solutions to this problem. Initial measurements indicate that wideangle scatter in the final focusing optics decreases the toral amount of power delivered to the target; additional test will be conducted to confirm this effect and determine its depen. dence on the configuration and finish quality of the final opxics. Smoothness criteria for the focal spot will be tested with the implementation of one-dimensional SSD on the Beamlet later this year.

\section{ACKNOWLEDXGENTS}

We would like to acknowledge the Beamlet operations team, whose dedicated efforts have made this work possible. C. Clay Widmayer performed the perturbation calculation of Figure 3b. The 16-level kinoform phase plate used in the tests was fabricated by Michael C. Rushford and Ian M. Thomas. This work was performed under the auspices of the U. S. Department of Energy by Lawrence Livermore National Laboratory under contract No. W-7405-ENG.48.

\section{REFERENCES}

1. J. D. Lindl, R. L. McCrory and E.M. Campbell, "Progress toward ignition and burn propagation in inertial confinement fusion," Physics Taday, 45, 32 (1992).

2. R. L. Berger, "Suppression of parametric instability by weakly incoherent laser beams," Phys. Rev. Lent. 65. 1207 (1990).

3. National Ignition Facility Conceprual Design Report, Volume 2; Design Basis and Requirements, p. 3-41, UCRL-PROP-117093 Vol 2, Livermore, CA, (1994).

4. S. Skupsky, R. W. Short, T. Kessler, R. S. Craxton, S. Letzring and I. M. Soures,"Improved laset beam uniformity using the angular distribution of frequency modulated light," J. Appl. Phys., 66, 3456 (1989).

5. S.N. Dixit, M. C. Rushford, I. M. Thomas and M. D. Perry. "Continuous contour phase plates for tailoring the focal plane irradiance profile," Proc. Soc. Photo.Opt. Instrum. Eng. 2633, 141 (1995).

6. A. C. Erlandson, M. D. Rotter, D. N. Frank and R. W. McCracken, ICF Quarterly Report, Lawrence Livermore National Laboratory, Livermore, CA, UCRL-LR-105821$95-1,5,18(1994)$.

7. J. T. Salmon, E. S. Bliss, J. L. Byrd, M. Feldman. M. A Kartz, J.S. Toeppen, B. Van Wonterghem and S. E. Winters, "An adaptive optics system for solid-state laser systems used is inertial confinement fusion." Proc. Soc. Photo-Opt. Instrum. Eng., 2633. 105 (1995). 
8. V. 1. Bespalov and V. L. Talanov, "Filamentary structure of light beams in nonlinear liquids," JETP Lett., 3, 307 (1960).

9. R. L. Carman, R. Y. Chiso and P, L. Kelley, " Observation of degenrate stimulated four-phocon interaction and fourwave parametric amplification," Phys. Rev. Lett., 17. 1281 (1966).

10. E. S. Bliss, D. R. Speck, J. F. Holzrichter, J. H. Erkkila and A. J. Glass,"Propagation of a high-intensity laser pulse with small-scale intensity modulation," Appl. Phys. Lett., 25, 448 (1974).

11.J. B. Trenholme, Laser Program Annual Repors, Lawrence Livermore National Laboratory, Livermore, CA, UCRL-50021-75, 237 (1975).

12. B. M. Van Wonterghem, J. R. Murray, J. H. Campbell, D. R. Speck, C. E. Barker, I. C. Smith, D. F. Browning and W. C. Behrendt, ICF Quarterly Report, Lawrence Livermore National Laboratory, Livermore, CA, UCRL-LR105821-95-1, 5, 1 (1994).

13.M. D. Rotter, R. W. McCracken, A. C. Erlandson and D. Brown, "Thermal recovery measurements on multisegment amplifiers," Proc. Soc. Photo-Opt. Instrum. Eng., 2633, 70 (1995). 


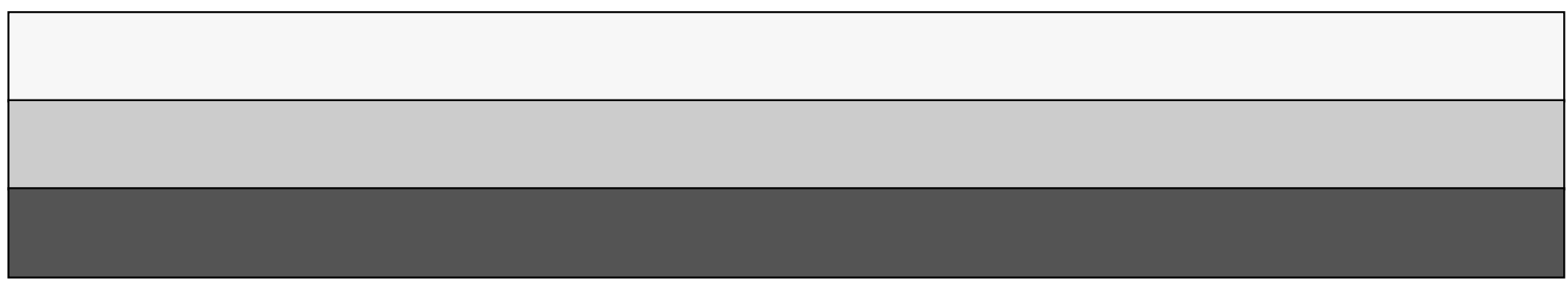

